Klaus G. Saur*

\title{
Wissenschaftliche Verlage - Versuch einer Prognose
}

https://doi.org/10.1515/bfp-2021-0026

Zusammenfassung: Die Emigration deutscher Verleger während der NS-Herrschaft führte zur Gründung neuer wissenschaftlicher Verlage in Großbritannien und den USA. Die Entwicklung des Verlagswesens wurde durch technische Neuerungen stark geprägt. Zunächst revolutionierte seit 1955 die Erfindung des Lichtsatzes die Druckbranche. Der Einsatz von Mikrofilmen und Mikrofiches ermöglichte neue Produkte wie das Deutsche Biographische Archiv. An ihre Stelle traten zunehmend CD-ROMund Datenbanken im Internet. Dieses ermöglichte OpenAccess-Publikationen, die vor allem im Bereich der Nachschlagewerke viele erfolgreiche Verlagsprodukte kannibalisierten (z.B. Brockhaus und Pschyrembel: Wikipedia). Auch das Selfpublishing bedeutet eine zunehmende Gefahr für die Verlage, vor allem seit der digitale Druck kostengünstige Kleinstauflagen ermöglicht. Dies hat zwar $\mathrm{zu}$ einer erheblichen Kostenreduzierung bei den Verlagen geführt, bietet aber auch Dienstleistern wie Books on demand eine Chance, an ihre Stelle zu treten. Die Umsätze auch bei den wissenschaftlichen Großverlagen gehen ohne Berücksichtigung der Zukäufe zurück. Die Prognose für die weitere Entwicklung der wissenschaftlichen Buchverlage ist schlecht, weil Verluste durch innovative Produkte wie E-Books und Hörbücher oder neue Serviceleistungen wie Fortbildungsveranstaltungen nicht ausgeglichen werden können. Man muss mit einem Rückgang von $15 \%$ rechnen, der viele Verlage gefährden wird. Einzige Ausnahme bilden die juristischen Verlage, die weiter mit Gewinn arbeiten.

Schlüsselwörter: Wissenschaftliche Verlage; Open Access; Digital Print; Printing on Demand

\section{The Academic Publisher - Attempting a Forecast}

Abstract: The emigration of German publishers during the Nazi regime resulted in new academic publishing activities to Britain and the USA. The development of the publishing industry was mainly driven by technical innovation. The invention of phototypesetting in 1955 revolutionised the

*Kontaktperson: Prof. Dr. h. c. mult. Klaus G. Saur, kgsaur@googlemail.com printing industry. Microfilming and microfiching made the development of new products such as the Deutsche Biographische Archiv, which were followed by CD-ROM and online databases, possible. Open access publications such as Wikipedia were able to cannibalise publishing products like Brockhaus and Pschyrembel. Self-publishing has created additional risks for publishers, since digital printing has reduced the costs for short run editions. Although, this has considerably reduced publisher costs, it has also allowed service providers such as books on demand a chance to replace them. The sales of large academic publishers are also declining if increases via the acquisition of newly bought publishing houses are disregarded. The prognosis for the future is serious. Losses cannot be compensated by innovative products such as e-books and audiobooks or new services like advanced training courses. Estimated losses of $15 \%$ will endanger many publishers. Only juridical publishing houses will operate at profit.

Keywords: Academic publisher; open access; digital printing; printing on demand

\section{Inhalt}

Ein Rückblick. . . . . . . . . . . . . . . . . . . . . . 209

2 Die Entwicklung des Lichtsatzes und seine Konsequenzen ..................... 209

3 Mikroeditionen ................... 209

4 Das Internet ...................... 210

5 Open Access . . . . . . . . . . . . . . . . . . 211

6 Amazon ... . . . . . . . . . . . . . . . . 212

7 Wikipedia................... 212

8 E-Books ....................... 213

9 Google oder Alphabet. . . . . . . . . . . . . . . . 213

10 Urheberrecht und VG WORT. . . . . . . . . . . . 213

11 Books on Demand . . . . . . . . . . . . . . 213

12 Export von Publikationen . . . . . . . . . . . . . 214

13 Anzeigenerlöse für Verlage . . . . . . . . . . . . 215

14 Titelproduktion ................... 215

15 Die Umsatzentwicklung der wissenschaftlichen

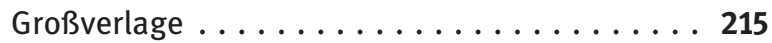

16 Innovative Produkte. . . . . . . . . . . . . . . 216

17 Wachstum für Verlage . . . . . . . . . . . . 216

18 Versuch einer Prognose für die nächsten Jahre . . 217 
19 Vorhersage für $2020 \ldots \ldots$. . . . . . . . . . . . 218

20 Ausblick für 2021 . . . . . . . . . . . . . . . . . . . 218

21 Anhang ...................... 218

22 Kommentar....................... 219

\section{Ein Rückblick}

Die ersten Wissenschaftsverlage wurden um 1500 gegründet. Die Oxford University Press, die Cambridge University Press, der Verlag E.J. Brill in Leiden, Benno Schwabe in Basel und Elsevier in Leiden, dann Amsterdam, entstanden aus privaten bzw. aus universitätseigenen Druckereien und verlegten von Anfang an Bücher, Serien und enzyklopädische Werke sowie Handbücher und monografische Publikationen in Papierform. Die wirtschaftliche Basis bei Oxford University Press und Cambridge University Press war damals das Bibelmonopol, das ihnen übertragen wurde. Alle diese Verlage existieren heute noch; Elsevier, 1713 geschlossen und 1880 neu gegründet, ist heute der weltweit größte Wissenschaftsverlag. Die Programme dieser Verlage sind im Prinzip vergleichbar, sowohl die wissenschaftlichen Gebiete als auch die Formen: Buch, Zeitschrift oder Serie, heute in digitaler oder gedruckter Version.

Die geniale Jahrtausenderfindung Johannes Gutenbergs, das Drucken mit der beweglichen Letter, war die Voraussetzung für diese Entwicklung. Bis 1933 gab es wissenschaftliche Verlage, die internationale Publikationen herausbrachten, nahezu ausschließlich in Deutschland, Großbritannien und den Niederlanden. Die Emigration deutscher Lektoren und Verleger ab dem Jahr 1933 ermöglichte es, dass in den USA wissenschaftliche Privatverlage gegründet wurden. Vor 1940 gab es in den USA eine Unmenge von sogenannten University Presses, die aber nicht wie die Oxford University Press und die Cambridge University Press im großen Maßstab internationale wissenschaftliche Literatur verlegten und verbreiteten, sondern wie der Name schon sagt - Druckereien einer Universität waren. Ihre Aufgabe bestand darin, die Bücher der an der Universität ansässigen Professoren zu verlegen. In der Praxis war es so: Viele Professoren versuchten ihre Bücher in Großbritannien, den Niederlanden oder in Deutschland zu publizieren. Wenn sie Absagen erhielten, war die hauseigene Druckerei immer noch gut genug, um das Buch herauszubringen; die University Presses in den USA waren immer Zuschussbetriebe. Durch die Emigration entstanden u. a. in den USA die Verlage Academic Press, der heute weitgehend zu Elsevier gehört, Interscience, der heute zu John Wiley gehört und die Grundlage für die wissenschaftlichen Publikationen bei dem bis dato nur als Schulbuchverlag tätigen Verlag John Wiley bedeutete. Viele mittlere und kleinere Verlage kamen auf diesem Wege dazu. In Großbritannien gründete Robert Maxwell den Verlag Pergamon Press, der heute ebenfalls zu Elsevier gehört.

\section{Die Entwicklung des Lichtsatzes und seine Konsequenzen}

Die Arbeit der Verlage mit der Herstellung von Büchern und Zeitschriften basierte rund 450 Jahre ausschließlich auf dem Prinzip von Gutenberg. Es kamen freilich weitere Verfahren hinzu, etwa der Flachdruck. Es kamen neue und preiswertere Bindeverfahren dazu, aber das Gesamtsystem beruhte immer noch auf Gutenberg. Eine entscheidende Veränderung ergab sich, als 1955 der Lichtsatz in den USA erfunden wurde. Das neue Prinzip war, dass Texte in elektrisch betriebene Schreibmaschinen eingegeben wurden, die dann direkt übertragen wurden, um von diesen Vorlagen zu drucken.

Bei der Produktion der Bücher änderte sich, zumindest für den Benutzer, kaum etwas. Es gab die gleichen Buchund Zeitschriftenausgaben wie bisher; aber die erste Konsequenz dieser neuen Entwicklung war, dass etwa 30000 Setzer, die noch in den 1950er-Jahren in Deutschland beschäftigt waren, auf Dauer arbeitslos wurden oder ihren Beruf wechseln mussten. Die Einführung des Lichtsatzes aber war auch die Voraussetzung dafür, dass später ein Internet geschaffen werden konnte, dass CD-ROM-Ausgaben, DVD-Ausgaben und Online-Daten-Banken hergestellt werden konnten. 1959 wurde die XEROX-Trockenkopie erfunden. Bis zu diesem Zeitpunkt kostete eine Fotokopie im Agfa-Nassverfahren eine D-Mark. Ein Betrag, der heute in etwa drei Euro entsprechen würde. Das bedeutete, dass es viel teurer war, 20 Seiten aus einem Buch zu kopieren, als das Buch zu kaufen. Durch die Erfindung der XEROX-Kopie fielen innerhalb kurzer Zeit die Kopierkosten pro Seite auf bis unter einen Pfennig. Dies bedeutete für die Verlage den massivsten Rückgang bei den von ihnen verlegten Lehrbüchern. Es war inzwischen preiswerter, ganze Bücher zu kopieren oder zumindest Teile, einzelne Kapitel, die der Student oder die Studentin jeweils benötigten, als die Originalwerke zu kaufen.

\section{Mikroeditionen}

1928 wurde im Schweizerischen Buchhandelsort Olten der Mikrofilm erfunden. Er wurde als Rollfilm in 18- oder $36 \mathrm{~mm}$-Fassung entwickelt. Das System wurde vor allem eingesetzt, um umfassende Datensammlungen oder Text- 
sammlungen zu verfilmen und sie auf diese Weise sicher zu erhalten. 1936 wurde der Mikrofiche im Postkartenformat entwickelt, der zwischen 96 und 420 Seiten auf einer Karte vereinigen konnte. Eine relativ starke Entwicklung nahm die Mikrofilmtechnik im Zweiten Weltkrieg, da die Mikrofilme in großem Maße zu Spionagezwecken eingesetzt wurden. Beginnend in den USA, dann aber auch in der Entwicklung bei vielen weiteren westlichen Staaten, wurde der Mikrofilm primär eingesetzt, um umfangreiche Zeitungsbestände zu verfilmen. So wurde in Deutschland beispielsweise beim Institut für Zeitungsforschung in Dortmund das Mikrofilm-Archiv der Deutschen Presse gegründet. In den 1960er-Jahren gingen mehr und mehr Fachverlage dazu über, diese Technik auch für kommerzielle Produkte einzusetzen. Große Datensammlungen wurden so verfilmt und kommerziell dem Bibliotheksmarkt angeboten. In den allermeisten Fällen handelte es sich um reine Wiedergaben vorhandener Textsammlungen oder Archivbestände.

1975 begann bei der Universität Marburg mit dem Fotoarchiv Marburg die Verfilmung des sogenannten Marburger Index, einer Bildsammlung zur Kunst- und Architekturgeschichte mit rund drei Millionen Aufnahmen, die in Marburg gesammelt worden waren. Hier wurden die Bilder nach geografischen Einheiten zusammengestellt, entsprechend veröffentlicht und durch den K. G. Saur Verlag weltweit vertrieben. Derselbe Verlag begann 1980 mit dem „Deutschen Biographischen Archiv“ die Verfilmung von biografischen Artikeln aus Lexika, bei denen aber mit dem Prinzip, Buch für Buch zu verfilmen, gebrochen wurde; stattdessen wurde die Reprokumulation angewandt, das heißt, das erste „Deutsche Biographische Archiv“ enthielt 450000 Artikel zu Persönlichkeiten aus dem deutschen Kultur- und Sprachraum bis 1900 zu etwa 220000 Personen; ausgewertet und reprografisch übernommen wurden alle Vorlagen aus insgesamt 450 Lexikon- und Enzyklopädie-Editionen. Dieses System bewährte sich und es wurde weiter entwickelt zum „Weltbiographischen Informationssystem“.

Einige weitere Verlage in Deutschland, den Niederlanden sowie in Großbritannien und in den USA verfilmten und editierten weitere Editionen auf Mikrofiche. In den 1970er- und 1980er-Jahren verursachten diese Produkte einen kommerziellen Umsatz auf dem Markt von jährlich rund 20 Millionen D-Mark. Dies entsprach rund zwei Promille des damaligen Buchmarktes. Mit der elektronischen Entwicklung der CD-ROM-Versionen, der DVD-Versionen und insbesondere wegen der massiven Erweiterungen der Internet-Datenbanken verloren die Mikrofiche-Editionen weitgehend ihre Bedeutung und stellen heute keinen nennenswerten Umsatzfaktor mehr dar. Für rund 20 Jahre waren sie aber auf dem Markt eine durchaus ernstzuneh- mende Größe, die auch eine wirtschaftliche Bedeutung erreichte. Sie ermöglichte eine Reihe von Editionen, die auf anderem Wege nicht realisierbar gewesen wären.

\section{Das Internet}

1968 startete ein Vorläufer des Internets - das Arpanet. Das System sah zunächst vor, die Kommunikation einiger weniger militärischer und akademischer Einrichtungen in den USA über Computer herzustellen. Bis 1990 war es in Betrieb, bevor es durch das technologisch verbesserte und grundsätzlich öffentlich zugängliche Internet abgelöst wurde. Anfänglich wurde die Idee der Computervernetzung aufgrund des geringen technischen Niveaus mehr oder weniger als Spielerei angesehen. Dies sollte sich schon im Laufe der späten 1970er- und der 1980er-Jahre massiv ändern. Zunächst kamen deutlich verbesserte digitale Datenträger heraus. 1985 die erste CD-ROM, die vergleichsweise große Datenmengen auf geringem Raum speichern konnte und die zunächst den Mikrofilm und den Mikrofiche verdrängte. Jedes neue Medium besaß nun eine kürzere Lebenszeit, denn schon 1991 kam die erste DVD heraus, die etwa die zehnfache Speicherkapazität bot und bessere Ergänzungsmöglichkeit zur Verfügung stellte. Der rasante Fortschritt der Computertechnologie schuf die Basis dafür, dass Zeitschriften parallel sowohl in Papierform wie in Online-Form verlegt werden konnten. Es blieb in den 1980er- und 1990er-Jahren auch vorwiegend so, dass der gleiche Verlag sowohl Papierausgabe wie die Online-Ausgabe betrieb und sie beide anbot. Inzwischen sind wohl 98 Prozent der wissenschaftlichen Zeitschriften elektronisch verfügbar, rund fünf Prozent ausschließlich elektronisch.

Aufgrund dieser technischen Entwicklung wurde es möglich, mit Open Access neue Produkte zu schaffen oder Produkte auf diesem Wege anzubieten. Diese Entwicklung wurde dadurch entscheidend beeinflusst, dass die wissenschaftlichen Verlage die Preise für ihre Zeitschriften seit den 1950er-Jahren bis 1995 in vielen Fällen um etwa 2000 - 4000 Prozent erhöht hatten. Die Bücherpreise waren im gleichen Zeitraum vielleicht um 100-250 Prozent gestiegen. Die Zeitschriftenpreise stiegen derart exorbitant, dass sie so gut wie jeden Bibliotheksetat sprengten. Inzwischen erscheinen rund 20000 Zeitschriften als OpenAccess-Veröffentlichungen. Das heißt, der Herausgeber bezahlt oder schafft die finanziellen Voraussetzungen, um die Dateneingabe zu finanzieren und die Veröffentlichungen dann seinem Publikum kostenlos online zur Verfügung zu stellen. Zug um Zug etabliert sich dieser Bereich, so dass mehr und mehr Informationen auf diesem Wege verbreitet werden. 


\section{Open Access}

1993 wurde durch eine Konferenz in Budapest der Begriff „Open Access“ weltweit bekannt gemacht. Darunter versteht man im Prinzip den kostenlosen Zugang für Benutzer auf elektronisch gespeicherte Daten und Texte. Man kann den Begriff aber auch weiter fassen. Er bedeutet dann kostenlose Information für Benutzer unabhängig davon, ob in elektronischer Form oder als gedruckte Buch- oder Zeitschriftenpublikation. Wikipedia ist heute das bekannteste Projekt weltweit, das hierunter fällt. Die Basis von Wikipedia ist, dass weltweit Daten von - wie auch immer freien Mitarbeitern erstellt oder aus Datenbanken kostenlos übernommen und der Öffentlichkeit kostenlos zur Verfügung gestellt werden. Die Kosten, die damit für den Vertreiber verbunden sind, werden durch finanzielle $\mathrm{Zu}$ schüsse von Großforschungsbereichen, Ministerien und Stiftungen sowie in einem erheblichen Maße auch von den Nutzern der Online-Enzyklopädie selbst gedeckt. Das größte und erfolgreichste Open-Access-Produkt in Papierform ist die „Apotheken-Umschau“, die in einem Verlag in der Nähe Münchens alle 14 Tage neu erscheint und über die Apotheken kostenlos an alle Interessenten verteilt wird. Der alle zwei Wochen erfolgende Vertrieb liegt bei über zehn Millionen Exemplaren und wird finanziert, indem die Apotheken für die Abgabe je 90 Cent pro Heft an den Verlag bezahlen; rund ein Drittel der Einnahmen erfolgt durch Anzeigen aus der Industrie, die in dieser Zeitschrift fast exklusiv inserieren.

Ursache für die öffentliche Open-Access-Diskussion die Anfang der 1990er-Jahre begann, war die Tatsache, dass die wissenschaftlichen Verlage, insbesondere die Großverlage Elsevier, Springer, Wiley, die Abonnementkosten innerhalb der letzten 50 Jahre um teilweise über 4000 Prozent erhöht hatten und ihre Monopolstellung bei Zeitschrifteninhalten erheblich ausnutzten. Der frühere Präsident der Max-Planck-Gesellschaft, Peter Gruss, argumentierte, dass die Wissenschaftler der Max-Planck-Gesellschaft, die etwa drei Prozent der Zahl der deutschen Wissenschaftler ausmachen würden, rund 30 Prozent der Artikel lieferten, die in internationalen Referate-Organen und Diensten zitiert werden; die Mitarbeiter der MaxPlanck-Gesellschaft würden die entsprechenden Aufsätze mit Mitteln ihres Arbeitgebers erstellen und diese satzfertig an Verlage übertragen, die diese nur noch in Zeitschriften abzudrucken oder in Online-Ausgaben überteuert zu verbreiten brauchten.

2010 hatte die Entwicklung einen Stand erreicht, dass schon rund 95 Prozent der naturwissenschaftlichen Zeitschriften parallel in gedruckter und in Online-Form angeboten wurden. Bei den Geisteswissenschaften handelte es sich um rund 70 Prozent Anteile. Peter Gruss äußerte deutlich, es sei unzumutbar, dass die Max-Planck-Gesellschaft die Aufwendungen für alle diese wissenschaftlichen Beiträge leisten und dafür dann noch extrem hohe Abonnementgebühren von häufig über 5000 Euro pro Abonnement an die entsprechenden Verlage zahlen müsse. Er empfahl dringend, Datenbanken aufzubauen, die gemeinnützig betrieben und diese Aufsätze in Online-Form kostenlos verbreiten würden. Er könne sich vorstellen, dass Aufsätze, die weiterhin in Fachzeitschriften - sowohl Papier wie online - erschienen, spätestens sechs Monate nach dem originären Erscheinen in den Datenbanken kostenlos verfügbar seien. Die Entwicklung nach 1993 begann zögerlich. Es wurden in den USA eine Reihe solcher Datenbanken aufgebaut, die von Großforschungsanlagen finanziell unterstützt wurden und Artikel überwiegend aus den Bereichen Medizin und Biologie auf diese Weise verbreiteten.

2003 fand die Berliner Open-Access-Konferenz statt, in der alle diese Ziele noch einmal deutlich formuliert und zahlreiche Anregungen für die Realisierung diskutiert wurden. 2004 standen dann rund 1000 Fachzeitschriften zur Verfügung, die Open Access verbreitet wurden. Bis 2010 erhöhte sich die Zahl auf 4000 Fachzeitschriften und heute stehen mehr als 20600 Zeitschriften online als Open-Access-Veröffentlichungen kostenlos für die Benutzer zur Verfügung. Man rechnet damit, dass es weltweit ungefähr 40000 wissenschaftliche Zeitschriften gibt, die bisher kommerziell vertrieben wurden, und dass davon 97 Prozent entweder auf Papier und online oder einige auch nur noch online angeboten werden. Man kann die Zahl 20000 Open-Access-Zeitschriften noch nicht in eine direkte Beziehung $\mathrm{zu}$ den vorhandenen kommerziellen Zeitschriften setzen, da zahlreiche kommerzielle Zeitschriften ein größeres Angebot an Seiten bieten als die rasch als Spezialorgane erscheinenden Open-Access-Zeitschriften. Auf jeden Fall sind aber die Open-Access-Produkte wesentlich schneller auf dem Markt und für die Wissenschaft zugänglich als die bei den kommerziellen Verlagen erscheinenden Artikel.

Fest steht, dass ein erheblicher Teil der Rückgänge der Umsätze wissenschaftlicher Verlage in den letzten fünf Jahren entscheidend darauf zurückzuführen ist, dass immer mehr Publikationen kostenlos über Open Access angeboten werden. Da die Zahl der Open-Access-Journale permanent steigt, wird es nur noch wenige Jahre benötigen, um etwa 50 Prozent der bisher kommerziell betriebenen Zeitschriftenbeiträge durch Open Access zu ersetzen. Aus der Entwicklung der Adressbuchverlage, der Wörterbuch- und Lexikon-Verlage wissen wir, dass die massive Gefahr für den Rückgang von Verkäufen dieser Produkte 
sowohl in Buch- wie in elektronischer Form vor allem dann einsetzte, wenn 50 Prozent der Inhalte in den kostenlos zugänglichen Datenbanken zur Verfügung standen.

Bei dem weltweit führenden „Klinischen Wörterbuch“ von Willibald Pschyrembel, das bei De Gruyter in Berlin erscheint, war die Situation so, dass in den ersten drei Jahren nach 2005, als dieses Wörterbuch komplett elektronisch angeboten wurde und man es in CD-ROM, DVD oder auch als Intranet-Lösung kaufen konnte, der Umsatz der elektronischen Umsätze stärker nach oben ging als die Umsatzrückgänge im Papierbereich. 2008 hatte sich insbesondere bei Wikipedia die Qualität der Daten auf dem Sektor Medizin derart erhöht, dass die Konkurrenz zum „Pschyrembel“ immer deutlicher wurde. Es begann der massive Rückgang des Verkaufs sowohl als Buch-als auch in elektronischer Form. Die Stanford University hat unlängst die sogenannte „Stanford Encyclopaedia of Philosophy“ freigeschaltet. Dieses Produkt geht weit über den Titel dieser Ankündigung hinaus. Es stellt eine enzyklopädische Gesamtinformation zu allen Humanwissenschaften dar und ist von einer ungewöhnlichen Qualität, die noch weit über das Niveau der Angaben bei Wikipedia hinausgeht; sie ist wesentlich gründlicher und informiert umfassender. Wären die „Brockhaus Enzyklopädie“ und die „Encyclopedia Britannica“ nicht schon seit längerer Zeit eingestellt, hätte es für diese Produkte das definitive Aus bedeutet. In immer kürzeren Abständen werden weitere Nachrichten publik, dass an der einen oder anderen Stelle von den unterschiedlichsten Organisationen weitere Texte online angeboten werden, die vorhandene Buch- und Zeitschriftentitel ersetzen oder zumindest reduzieren werden.

\section{Amazon}

Amazon wurde 2002 in den USA gegründet und breitete sich innerhalb kürzester Zeit weltweit aus. Es ist heute das größte Versandhandelsunternehmen, das es überhaupt gibt. Schwerpunkt der Arbeit liegt unter anderem im Buchversand sowie in weiteren Medien, die verschickt werden. Amazon hat eine gigantische Macht erworben und steigerte seine Umsätze in massiver Weise. Auf der einen Seite bedeutet dies einen starken Einbruch für die Buchhandlungen, da immer mehr Bestellungen von Privatkunden über Amazon abgewickelt und nicht mehr von Buchhandlungen ausgeführt werden. Amazon hat mehrere Versuche unternommen, auch verlegerisch tätig zu werden. Aufgrund der Angebotstechnik müsste es möglich sein, Buchprodukte zu schaffen, die mehr oder weniger exklusiv über Amazon angeboten werden. Bis jetzt sind diese Versuche gescheitert. Man muss davon ausgehen, dass Amazon sich erheblich weiterentwickelt und gewaltige Finanzbeträge in diese Entwicklungen steckt. Es ist auf jeden Fall zu erwarten, dass Amazon in Zukunft Bücher sowohl in gedruckter wie in elektronischer Form vertreiben wird. Dieses Angebot würde nicht auf die Belletristik beschränkt sein, sondern nach bereits vorhandenen Plänen massiv in den wissenschaftlichen Informationsbereich hineinragen. Es ist noch offen, wann diese Projekte realisiert werden. Vorstellbar ist, dass im Laufe der nächsten zwei Jahrzehnte ein erheblicher Anteil der Verlagsprodukte durch Amazon-Angebote entweder reduziert oder verdrängt wird.

Amazon ist heute in vielen Fällen der wichtigste Kunde der Verlage oder zumindest bei den größten Abnehmern vertreten. Zahlreiche Verlage erzielen schon mehr als zehn Prozent ihres Umsatzes mit dem Handelsriesen. Der Druck auf Handelsspannen von Amazon ist hoch und die meisten Lieferungen an Amazon werden nur mit einem Rabatt realisiert, der 10 bis 20 Prozent über den üblichen Handelsrabatten liegt. Das bedeutet für die Verlage im Prinzip eine Verschlechterung ihres Betriebsergebnisses um mindestens 2 Prozent.

\section{Wikipedia}

Wikipedia, 1998 in den USA gegründet, erweiterte seine Plattform auf dem deutsch- und französischsprachigen Bereich ab 2001 und ist inzwischen weltweit verbreitet. Der Chef von Wikipedia Deutschland kam 2003 nach Mannheim, stellte sich hinter die Büste von Friedrich Arnold Brockhaus, dem Gründer des Verlages Brockhaus, und erhob seine Hände zum Victory-Zeichen. Ich bekam ein Foto von dieser Situation und empfand es zunächst als Unverschämtheit und hielt es für mehr als illusionär zu glauben, dass Wikipedia Brockhaus besiegen könne. F. A. Brockhaus, zum damaligen Zeitpunkt in Mannheim angesiedelt, hatte in den 1980er-Jahren mit der 18. Auflage der „Brockhaus Enzyklopädie“ den wirtschaftlich größten Erfolg in der Verlagsgeschichte erzielt. Das Markenlexikon galt neben der „Encyclopedia Britannica“ schon seit vielen Jahren als die weltweit beste Enzyklopädie der neueren Zeit. $\mathrm{Zu}$ Beginn des 21. Jahrhunderts waren die Daten, die Wikipedia geboten hat, in vielen Fällen nicht nur schlechter, sondern auch weniger aktuell. Dies änderte sich binnen kurzer Zeit dramatisch und führte dazu, dass Brockhaus 2005, im Jahre des 200-jährigen Jubiläums (das noch ungewöhnlich aufwändig gefeiert worden war) seinem Untergang deutlich entgegen ging. Bereits 2009 wurde Brockhaus an Bertelsmann verkauft, wobei Bertelsmann dies mehr auf Wunsch des Inhabers Reinhard Mohn tat als aus 
wirtschaftlichen Erwägungen. Es stellte sich bald heraus, dass die Entwicklung - weg vom kostenpflichtigen Printlexikon hin zum für den Nutzer kostenfreien Onlinelexikon - nicht mehr aufzuhalten war. 2014 wurde die Marke Brockhaus bei Bertelsmann endgültig stillgelegt und ruht seitdem. Dies ist ein erster prominenter Fall, dass durch Daten, die von Wikipedia für die Benutzer kostenlos verbreitet wurden, eine so etablierte Verlagsmarke wie Brockhaus vom Markt verschwunden ist.

Die Entwicklung ging weiter. Der „Duden“ wurde ebenfalls vom alten Inhaber-Verlag verkauft. Er erlebte bei Cornelsen einen massiven Rückgang. Heute hat er nur noch einen Bruchteil der Umsätze, die noch vor zehn Jahren galten. Der Verlag Langenscheidt, der bis in die 1990erJahre der weltweit größte Verlag für zweisprachige Wörterbücher war, ist inzwischen auch verkauft worden. Eine Hamburger Lottofamilie erhält ihn weiter und erreicht heute noch knapp 10 Prozent der Umsätze, der vor 20 Jahren erreicht wurden.

Wir müssen davon ausgehen, dass z.B. Wikipedia seine Daten permanent verbessert, was schon jetzt dazu geführt hat, dass der berühmte „Pschyrembel“, der in mehr als 270 Auflagen erschien und das erfolgreichste medizinische Wörterbuch der Welt war, nicht mehr gehalten werden kann, weil die Angaben in Wikipedia inzwischen wohl nur noch fünf Prozent schlechter sind als die Angaben im „Pschyrembel“, jedoch kostenlos zugänglich sind, so dass die Kunden des „Pschyrembel“ mehr und mehr zur Nutzung von Wikipedia übergehen.

\section{E-Books}

Inzwischen geben die meisten Verlage ihre belletristischen wie ihre wissenschaftlichen Buchproduktionen parallel sowohl in Papierform wie in E-Book-Form heraus. Der Anteil der E-Books am Gesamtumsatz ist in Deutschland im Jahr 2018 von 3,8 auf 4,5 Prozent gestiegen, das heißt, er macht nach wie vor einen vergleichsweise geringen Anteil aus. In vielen Fällen wäre es in der Praxis so, dass die Gesamtkosten der Verlage entsprechend niedriger und rentabler wären, wenn ausschließlich gedruckte Bücher verlegt würden. Doch es gibt keinen Autor mehr, der bei einem neuen Verlagsvertrag darauf verzichtet, dass seine Publikation auch als E-Book erscheint. Die Zukunft des EBooks ist noch völlig offen. Es kann bei den bisherigen Formen wie Kindle bei der Verbreitung bleiben, es wird aber völlig neue Formen geben, von denen wir heute noch sehr wenig wissen. Denn auch über Telefone, über Uhren und über andere Datenträger können Informationen verbreitet oder gespeichert werden. Faktum ist: Der Anteil der elektronisch erzeugten Daten und deren Verbreitung werden erheblich zunehmen und dies in den meisten Fällen zu Lasten papiergebundener Ausgabeformen.

\section{Google oder Alphabet}

Google ist im Augenblick im Begriff, sich in Alphabet umzubenennen, um seinen Gesamtanspruch auf die komplette Informationswelt deutlich zu machen. Schon heute vermittelt Google eine Unzahl von Daten, die für die Endabnehmer kostenlos zugänglich sind. Die Finanzierung erfolgt über Anzeigenerträge, die sich bei Google immer stärker entwickeln. Ein Ergebnis dieser Tätigkeiten ist, dass eine Reihe von bisher in gedruckter oder auch in elektronischer Form erscheinender Adressbücher bei kommerziellen Verlagen eingestellt werden musste. Es stellt sich immer mehr heraus, dass man umfassende Informationen zu Adressen, aber auch zu weiteren Daten bei Google kostenlos abrufen kann, ohne dass dafür noch irgendwelche Aufträge gegen Rechnung erteilt werden müssen.

\section{Urheberrecht und VG WORT}

Das Urheberrecht entwickelt sich ebenfalls in weiten Bereichen nachteilig für Verlage und Inhaber von Urheberrechten. Auf der einen Seite gibt es heute durch die neue Rechtsprechung wesentlich mehr Möglichkeiten, dass Bibliotheken Texte von prinzipiell geschützten Werken aufnehmen und kostenlos verbreiten. Auf der anderen Seite ist das Urheberrecht in der heutigen Form nicht ausreichend in der Lage, Rechtsbrüche zu verhindern. Es wird immer schwieriger zu ermitteln, wo welche Datenbanken oder Datenbestände illegal heruntergeladen oder entsprechend weiterverbreitet werden. In den meisten Fällen sind die Kosten für derartige komplizierte Untersuchungen für den Urheber, wenn er sie in Auftrag gibt, wesentlich höher als das, was er je als Schadensersatzsummen bekommen könnte.

\section{Books on Demand}

Bis 1995 hat die Druckmaschinenindustrie immer mehr Maschinen, Anlagen und Systeme entwickelt, die Bücher in höchsten Auflagen von über 10000 immer schneller und preiswerter herstellen konnten. Sie hatte aber dabei außer Acht gelassen, dass 98 Prozent aller Bücher in Auflagen von weniger als 10000 erscheinen, und dass immer mehr Bücher herauskommen, die weder 1000 noch 500 
als Verkaufsauflage erreichen können. Aus diesem Grund wurden nach 1995 Systeme entwickelt, die es ermöglichen, dass heute beispielsweise ein Buch in einer Auflage von 100 Exemplaren pro Stück preiswerter hergestellt werden kann als vor Jahren noch eine 500-er Auflage. Daraus entstand die Idee „Books on Demand“, die heute in Deutschland noch führend in einer Firma gleichen Namens realisiert wird. Es gibt weitere Anbieter, aber Marktführer ist die Firma Books on Demand, Norderstedt. 2019 hat sie rund 400000 Titel neu angekündigt.

Books-on-Demand-Bücher unterscheiden zwischen drei Bereichen. Der erste Bereich sind Privatdrucke, der zweite wissenschaftliche oder auch Fachbücher in kleinsten Auflagen, der dritte Bereich ist die Bereitstellung von Büchern, die bei den Verlagen - insbesondere bei den wissenschaftlichen Verlagen - in den letzten 250 Jahren erschienen sind.

Die erste Gruppe betrifft private Kunden, die zunächst einmal davon ausgehen, dass lyrische Bemühungen unzufriedener Hausfrauen oder Hausmänner unbedingt gedruckt werden müssen und zu einem Geburtstag an alle Gäste verteilt werden. Hier können die Autorin, der Ehemann oder die Kinder der Autorinnen oder Autoren einen Auftrag nach Norderstedt geben, dass sie beispielsweise 100 Exemplare in Schmuckausstattung von dem Text bestellen. Books on Demand in Norderstedt bietet an, dass diese Titel auf dem Markt angeboten werden. Je nach Interesse des Autors oder des Empfängers kann dies umgesetzt werden. Es gibt auch Fälle, in denen beispielsweise Kriminalromane, die klassische Verlage ablehnten, auf diese Weise bekannt und in einer Auflage von 10000 Exemplaren und mehr verbreitet wurden.

Der zweite Bereich setzt sich aus wissenschaftlichen Publikationen zusammen, die sehr speziell sind. Die bisherige Praxis war dabei so, dass der Herausgeber, das Institut oder die wissenschaftlichen Gesellschafter zum Verlag gegangen sind und erklärt haben: „Wir haben hier zwei neue Texte von je 250 Seiten, sehr bedeutend, und wir möchten sie gerne verlegt haben“. Der Verlag sagt: „Wunderbar, können wir gerne machen. Wir benötigen pro Band einen Druckkostenzuschuss von 4000 Euro“. Dann erklärt der potenzielle Auftraggeber, dass er nicht verstehen könne, dass bei Books on Demand das zweimal 900 Euro kostet und, wenn mehr als 50 Exemplare verkauft würden, bekomme er das Geld sogar ganz - oder noch mehr - zurück. Dann erklärt der Verlag: „Ja, dort fehle die verlegerische Betreuung“. Doch bei derartigen Spezialtiteln, die in kleinsten Auflagen erscheinen, bietet auch der klassische Verlag keine große verlegerische Betreuung. Das heißt, dass mehr und mehr Autoren und Institutionen dazu übergehen, solche Spezialtitel bei Books on Demand zunächst auf eigene Rechnung herstellen zu lassen. Doch dieser Betrag bleibt gering und kann auch in vielen Fällen zurückerstattet werden. Es gibt auch Fälle, wo Verlage selber erkennen und sagen: „Von diesem Buch können wir nicht mehr als 80 Stück verkaufen, also bestellen wir jetzt eine Books-on-Demand-Auflage in Höhe von 40 und lassen sofort weitere herstellen, wenn diese 40 verkauft sind“. Dies kann und wird ein Geschäft der Verlage bleiben, aber mit sehr begrenzten Umsätzen.

Der dritte Bereich betrifft die Verlage sehr intensiv. Wenn ein Verlag, unabhängig davon, ob vor 10, 20 oder 50 Jahren, eine Publikation verlegt hat und nicht bereit ist oder aus wirtschaftlichen Gründen nicht in der Lage ist, eine Neuauflage $\mathrm{zu}$ veranstalten, fallen im Prinzip die Rechte an den Autor zurück. Um dies zu umgehen, kann der Verlag nun bei Books on Demand einen Titel bestellen, beispielsweise eine bedeutende Monografie aus dem Jahr 1920, es wird bei Books on Demand angenommen, und sobald eine Bestellung eingeht, digitalisiert und ein Exemplar hergestellt wird. Damit bleiben die Rechte beim Verlag. Von Books on Demand oder auch von den Verlagen können diese Titel dann wieder als Information dem internationalen Buchhandel und den Bibliotheken zugeleitet werden. Die Konsequenz für die Zukunft der Verlage ist hier, dass auf der einen Seite Verlage dieses System benutzen können, um sich damit Rechte zu wahren und Objekte anzubieten, die sich seit vielen Jahren nicht mehr verkaufen ließen. Auf der anderen Seite wird dieses System dazu führen, dass eine Reihe von Publikationen, die bisher verbunden mit hohen Zuschüssen an Verlage gegeben wurden, nicht mehr einer Verlagsmitwirkung bedürfen. Inzwischen sind 4 Millionen Titel bei Books on Demand angekündigt bzw. lieferbar und können bei Bedarf auch in einem Stück hergestellt werden. Die Verlage erzielen dadurch beachtlich mehr Umsätze, da sie eine Lizenzgebühr für jeden Titel bekommen, der beispielsweise vor 200 Jahren erschienen ist und jetzt on Demand bestellt wird.

\section{Export von Publikationen}

Der Export deutscher wissenschaftlicher Publikationen ist in den letzten Jahren relativ stabil geblieben. Während der Umsatz der Verlage im Jahr 2019 um 1,3 Prozent gestiegen ist, sind die Exportzahlen um 2,7 Prozent höher, das heißt, der Binnenmarkt ist auf 1 Prozent gesunken und nur der Exportmarkt ist positiv. Rückläufig im Export sind insbesondere die Märkte Japan, Österreich und Schweiz. Stabil sind USA mit hohem wissenschaftlichen Umsatzbereich sowie Polen, Tschechien, Russland, Südkorea und China. 


\section{Anzeigenerlöse für Verlage}

In den 1970er-Jahren machten die Anzeigenerlöse in den Fachzeitschriften der Verlage noch einen erheblichen Umsatzanteil aus. Bei Verlagen wie Hanser, VDI, Georg Thieme, auch Springer betrugen diese Umsätze in vielen Fällen mehr als 10 Prozent der Gesamterlöse und trugen zum Gewinnergebnis noch überproportional bei. Heute gibt es kaum noch Industrieanzeigen. Die Werbung läuft wesentlich stärker über das Fernsehen oder über Zeitschriften, die das Endpublikum wie die „Apotheken-Umschau“ erreichen. Das heißt, die Anzeigenerlöse sind um mehr als 90 Prozent gesunken und stellen heute keinen wesentlichen Beitrag mehr für die Erträge dar. Das gilt auch für den Deutschen Apotheker Verlag in Stuttgart, zu dem die Wissenschaftliche Verlagsgesellschaft, der Franz Steiner Verlag und der S. Hirzel Verlag gehören. Inzwischen wurden sechs weitere Verlage hinzugekauft, welche die Basis des Verlags erweitert haben, und der Gesamtumsatz der Gruppe ist in den letzten Jahren um etwa 30 Prozent gestiegen. Aber auch hier gilt, dass die Summe der Umsätze der bisher zugehörigen Verlage plus der neuen vor zehn Jahren insgesamt höher war, als sie der Verlag jetzt erreicht.

\section{Titelproduktion}

2019 erschienen insgesamt 78746 Titel in den Verlagen der Bundesrepublik Deutschland. Davon waren Erstauflagen 70395.

Tab. 1: Titelproduktion für die Jahre 2010-2019

\begin{tabular}{rrr}
\hline Jahr & Neuerscheinungen insgesamt & davon Erstauflagen \\
\hline 2010 & 95838 & 84351 \\
2011 & 96273 & 82048 \\
2012 & 91100 & 79860 \\
2013 & 93600 & 81919 \\
2014 & 87134 & 73863 \\
2015 & 89506 & 76547 \\
2016 & 85486 & 72820 \\
2017 & 82636 & 72499 \\
2018 & 79916 & 71548 \\
2019 & 78746 & 70395 \\
\hline
\end{tabular}

Die höchste Zahl wurde 2011 erreicht mit 96273 . Seit 2015 geht es kontinuierlich nach unten und liegt 2019 bei 78746.
Tab. 2: Umsatzentwicklung nach Geschäftsarten 2015-2019 (Veränderungen zum Vorjahr in Prozent)

\begin{tabular}{lrrrrr}
\hline & $\mathbf{2 0 1 5}$ & $\mathbf{2 0 1 6}$ & $\mathbf{2 0 1 7}$ & $\mathbf{2 0 1 8}$ & $\mathbf{2 0 1 9}$ \\
\hline Bücher & $-4,0$ & $+0,7$ & $-0,1$ & $-0,4$ & $+0,8$ \\
Zeitschriften & $+0,2$ & $-1,1$ & $+2,1$ & $+1,7$ & $-13,4$ \\
$\begin{array}{l}\text { Online- } \\
\text { Dienste }\end{array}$ & $+14,3$ & $+14,7$ & $+12,7$ & $+14,8$ & $+33,2$ \\
$\begin{array}{l}\text { Sonstige } \\
\text { Waren }\end{array}$ & $+8,1$ & $+1,2$ & $+9,2$ & $+1,7$ & $+3,7$ \\
Nebenrechte & $-5,2$ & $+7,2$ & $+0,7$ & $+5,5$ & $+5,4$ \\
Anzeigen & $-0,2$ & $+2,5$ & $+2,4$ & $-4,9$ & $-6,6$ \\
\hline Insgesamt & $-2,0$ & $+1,8$ & $+1,7$ & $+0,2$ & $-0,5$ \\
\hline
\end{tabular}

Der Umsatz der deutschen Verlage stagniert seit 2015. Die einzigen Gruppen die steigen sind die Online-Dienste und die sonstigen Waren. Online-Dienste sind die Erlöse aus Open-Access-Verkäufen bzw. Online-Verkäufen und Internet-Verkäufen oder Lizenzen, die erzielt werden, wenn ein Verlag Inhaltsrechte zur weiteren Verwertung im OnlineBereich an einen anderen Verlag gibt.

Hier war die stärkste Entwicklung 2019, wo es eine Zunahme von 33,2 Prozent gab, während die Zeitschriften selbst um 13,4 Prozent zurückgegangen sind. Unter Sonstigen Waren versteht man Formulare wie beispielsweise bei Georg Thieme, dem größten medizinischen Verlag, der inzwischen das Formulargeschäft bei den deutschen Krankenhäusern weitgehend komplett beherrscht. $\mathrm{Zu}$ der Gruppe gehören Seminare, Fortbildungsveranstaltungen, Datenbankgeräte oder auch psychologische Tests bei Psychologie-Verlagen. Insgesamt ist das Ergebnis stagnierend und damit im Prinzip rückläufig.

\section{Die Umsatzentwicklung der wissenschaftlichen Großverlage}

Es gibt heute nach den zahlreichen Konzentrationen und Zukäufen nur noch vier wissenschaftliche Großverlage, die Umsätze von insgesamt über einer Milliarde Euro erzielen, wobei der Springer-Verlag Berlin, Heidelberg, New York, diesen Betrag auch nur dadurch erzielt, dass er mit den wissenschaftlichen Verlagen von Holtzbrinck zusammengelegt wurde und dadurch „Nature“ als eine der weltweit wichtigsten Zeitschriften zu Springer gekommen ist. Der Springer Verlag hatte im Jahr 2013 einen Umsatzrückgang von 1,8 Prozent. Der Gesamtrückgang der Verlagsprodukte Buch und Zeitschriften in Papier und online ist noch stärker, denn es wurden diverse Projekte aufgekauft, die in dem Umsatz enthalten sind. 
Reed-Elsevier hatte im Jahr 2013 zum ersten Mal seit 1949 einen Umsatzrückgang von 4 Prozent. Bei den einzelnen Sparten sind die Entwicklungen unterschiedlich: bei Risk and Business Information 3 Prozent Rückgang, bei Legal 11 Prozent Rückgang, bei STM 4 Prozent Rückgang. Lediglich bei Ausstellungen und Messen stieg der Umsatz um 3 Prozent. Das heißt, dass die Umsätze Buch und Zeitschrift sowie Gesamtinformation überall rückläufig sind und durch andere Produkte wie in diesem Fall Messen und Ausstellungen, teilweise noch ausgeglichen werden können.

Bei Wolters Kluwer stiegen die Umsätze von 3,5 auf 3,6 Millionen Dollar minimal an, aber auch hier ist der Anstieg nur auf Zukäufe zurückzuführen und nicht auf vorhandene Verlagsprojekte. Bei John Wiley wird vermutet, dass der Umsatzrückgang im Jahr 2014 rund 5 Prozent betrug. Es wurde inzwischen eine Arbeitsplatzreduzierung von 800 Mitarbeitern eingeleitet.

\section{Innovative Produkte}

Die wichtigste Innovation der letzten Jahrzehnte, die vor allem belletristischen Verlagen und dem Buchhandel insgesamt neue Umsätze und Erträge verschafften, ist das Hörbuch.

1997 wurde der Hörverlag München gegründet. Claudia Baumhöver, die Initiatorin des Verlags, entwickelte ein Konzept für einen Hörverlag nach dem Vorbild des Deutschen Taschenbuch Verlags, der 1950 gegründet wurde. Zunächst erfand sie das Hörbuch neu. Denn schon seit über 100 Jahren gab es Kassetten und Tonbänder, die Texte aufgenommen hatten, die aber primär von blinden, alten und kranken Personen genutzt wurden und eine vergleichsweise geringe Verbreitung fanden. Am stärksten entwickelt war das Angebot in Marburg bei der Deutschen Blindenanstalt, die derartige Programme für viele blinde Kunden entwickelte. Claudia Baumhöver brachte zunächst neun Verlage zusammen, darunter Suhrkamp, Hanser, Kiepenheuer \& Witsch und andere, um die Rechte dieser Verlage für erfolgreiche Buchausgaben nun um Hörbücher $\mathrm{zu}$ multiplizieren und $\mathrm{zu}$ erweitern. Dieses Konzept ging auf. Nach Gründung des Verlags 1997 wurde 2010 ein Umsatz von 25 Millionen Euro mit einem außerordentlich hohen Gewinnergebnis erreicht. Die Umsätze der Hörbücher erreichten bis zu 4,2 Prozent Anteil am Buchhandelsumsatz und lagen bis zum vergangenen Jahr höher als der Anteil der E-Books. Eine Folge der Entwicklung des Hörverlags war, dass auch andere Verlage diese Idee übernahmen und selbst Hörbücher produzierten und damit weniger Rechte an den Hörverlag abgaben. Da- durch ging der Umsatz des Hörverlags zurück, der Gewinn ging ebenfalls zurück und vor drei Jahren wurde dieser Verlag an Bertelsmann verkauft und mit Random-Hörbücher, die jetzt gemeinsam der Marktführer sind, zusammengelegt.

Weitere innovative Entwicklungen ergaben sich bei wissenschaftlichen Verlagen, indem beispielsweise ein großer medizinischer Verlag Abrechnungssysteme für Ärzte, Abrechnungstabellen und ähnliche Hilfsmittel für Ärzte entwickelte. Hinzu kamen große Veranstaltungen, Seminare, Kongresse und Kolloquien. Selbst ein vergleichsweise kleiner Verlag wie Meiner in Hamburg mit der berühmten „Philosophischen Bibliothek“ bietet inzwischen philosophische Seminare und Kongresse an, die gut besucht werden. Dies alles führt zu neuen Erträgen bei den Verlagen, aber gleicht nur einen Teil der Reduktionen auf dem klassischen Markt aus. Denn im Grundsatz gilt: Ein wissenschaftlicher Verlag hat heute einen Rückgang von 4 bis 5 Prozent im Jahr zu verkraften; er ist in einigen Fällen in der Lage, durch neue Systeme und Produkte etwa die Hälfte davon auszugleichen, so dass der Rückgang nur 2 bis 3 Prozent beträgt. Für viele Verlage bleibt es bei Rückgängen, und ein Verlag bezeichnet sich heute schon als erfolgreich, wenn er über mehrere Jahre seinen Umsatz in etwa halten konnte.

\section{Wachstum für Verlage}

Gibt es heute überhaupt noch ein Wachstum? Es verteilt sich auf ganz wenige Verlagsformen. Der Verlag Wort \& Bild in Baierbrunn bringt seit den frühen 1990er-Jahren die „Apotheken-Umschau“ und inzwischen neun weitere Zeitschriften heraus, die im Prinzip nach dem Open-AccessSystem laufen. Von der „Apotheken-Umschau“ werden wie schon angesprochen - alle zwei Wochen 9,5 Millionen Exemplare an die Apotheken in Deutschland, Österreich und Schweiz geliefert; die Apotheken zahlen dafür 90 Cent pro Heft und geben es kostenlos an ihre Kunden ab. Durch die massive Fernsehwerbung des Verlags wird permanent darauf hingewiesen: „Neu - in Ihrer Apotheke, kostenlos!“ Keine Apotheke kann es sich leisten, die Zeitschrift nicht kostenlos abzugeben, aber die Apotheker gehen auch davon aus, dass die Kunden, die wegen der Zeitschrift in die Apotheke kommen, in den meisten Fällen Produkte kaufen, so dass es für sie doch eine vergleichsweise gut eingesetzte Werbeaktion ist.

Der Landwirtschaftsverlag in Hiltrup in der Nähe von Münster hat vor einigen Jahren die Zeitschrift „Landlust“ gegründet. Er eröffnete damit ein neues Themenfeld, das zu Millionenerfolgen führte. Inzwischen gibt es eine Rei- 
he weiterer Zeitschriften, die mehr Auflagenzuwachs haben, als der Rückgang von „Spiegel“, „Stern“, „Focus“ ausmachen. Das heißt, der Umsatz mit den Unterhaltungszeitschriften im Handel ist nicht gesunken, sondern er verändert sich massiv. Das, was weniger an „Focus“ oder „Spiegel“ verkauft wird, gleicht sich durch „Landlust“, „Landliebe“ und ähnliche Publikationen aus.

Im Prinzip ist heute für Wissenschaftsverlage Wachstum nur noch durch Zukauf möglich. Der Verlag De Gruyter in Berlin macht aktuell einen Umsatz von etwas über 70 Millionen und hat damit gegenüber 2006 seinen Umsatz in etwa verdoppelt. Um das zu erreichen, wurden die Verlage K.G. Saur mit den angeschlossenen Verlagen Niemeyer, Francke und Zeller sowie dem Akademie-Verlag und dem Oldenbourg Verlag gekauft. Addiert man die Umsätze dieser Verlage, die 2005 noch selbstständig waren, kommt man auf einen Betrag von rund 58 Millionen Euro; das heißt, dass die Gesamtsumme der Umsätze dieser Verlage von 58 auf etwa 50-51 Millionen Euro gesunken ist. Die Einheit De Gruyter ist zwar größer geworden, doch der Gesamtumsatz in der Branche hat entsprechend abgenommen.

Der Deutsche Apotheker Verlag in Stuttgart, zu dem früher schon die Wissenschaftliche Verlagsgesellschaft, der Franz Steiner Verlag und der S. Hirzel Verlag gehörten, hat inzwischen fünf weitere Verlage hinzugekauft, die die Basis des Verlages erweitert haben. Doch ist dabei kein größerer Umsatz für den Markt entstanden.

\section{Versuch einer Prognose für die nächsten Jahre}

Prognosen haben leider fast immer die Eigenschaft, falsch zu sein. Es ist nicht möglich, präzise Vorhersagen abzugeben, ob und in welcher Form neue Produkte geschaffen werden. Es war zum Beispiel nicht vorhersehbar, dass 1998 in den USA, bzw. 2001 in Deutschland Wikipedia gegründet wird und innerhalb weniger Jahre ein Datenpotenzial anbietet, das eine Reihe der etabliertesten Verlage, insbesondere auf dem Sektor Lexikon, Wörterbuch, Enzyklopädie, zunächst erheblich reduzierte und dann mehr oder weniger ersetzte. Es war ebenso unvorhersehbar, dass aus den ersten Ansätzen und Überlegungen zum Thema Open Access ab 1993 tatsächlich eine so folgenreiche neue Entwicklung kommen werde. Die massive Kostensenkung für kleine Auflagen, die seit etwa 2005 in der Praxis Anwendung gefunden hatte, war ebenfalls von den meisten Experten in keiner Weise in diesem Umfang vorhergesehen worden.
Folgende Entwicklungen kann man aber mit großer Wahrscheinlichkeit näher beziffern oder benennen:

1) Fest steht, dass die Open-Access-Entwicklung massiv voranschreitet und in den nächsten zehn Jahren mehr als 50 Prozent der wissenschaftlichen Inhalte von Zeitschriften durch Open Access entweder ersetzt oder zumindest in Konkurrenz gebracht wird.

2) Die Erwerbungsetats sowohl der wissenschaftlichen wie der Öffentlichen Bibliotheken bleiben deshalb weiter unter erheblichem Druck, weil die Erwerbungsmittel immer mehr für elektronische, online zugängliche Materialien verausgabt werden und sich von daher die Beträge für den Ankauf von analogen Büchern und Zeitschriften reduzieren.

3) Die Bereitschaft öffentlicher Institutionen, elektronische Informationen verlagsunabhängig zu fördern und durch Verlage hergestellte Produkte nicht mehr oder nur noch in wesentlich geringerem Maße zu unterstützen, nimmt zu.

4) Die Verlagskonzentration wird weiter zunehmen. In den nächsten Jahren werden einige Verlage zu dem Ergebnis kommen, dass sie unabhängig voneinander nicht mehr operieren können und sie sich einem größeren Verbund anschließen müssen. Ebenso wird die Zahl der Liquidationen von Verlagsunternehmen zunehmen, da die dann noch vorhandene Basis nicht mehr ausreicht, um für eine Übernahme interessant zu werden.

5) Die weltweite Entwicklung des Urheberrechts läuft im Prinzip gegen die Interessen der Verlage und erleichtert den Benutzern immer mehr, Daten elektronisch zu übernehmen, ohne dass eine Gebühr dafür bezahlt wird. Auch die unzureichende Möglichkeit, Rechtsverstöße zu ahnden, bringt extreme Schwierigkeiten und vor allem Aufwendungen, die in keinem Verhältnis zu dem dann möglichen Nutzen von Entschädigungen stehen.

6) Es wird weiterhin einen Markt für gedruckte Publikationen geben. Wir erleben im Augenblick wieder eine Zunahme der Ausleihe von Büchern bei Bibliotheken sowohl in Deutschland, aber auch in den USA. Wir erleben, dass die größte britische Buchhandelskette alle Kindle-Geräte aus ihren Lagern entfernt und durch gut verkäufliche Bücher ersetzt, und wir haben nach wie vor eine große Zahl von interessierten Lesern, die ihre Bücher in gedruckter Form haben möchten und nur so konsumieren. Wissenschaftliche Literatur kann weiter existieren, wenn sie extrem originäre Werte enthält. Ein Pschyrembel-Wörterbuch hat kommerziell keine Zukunftschance, denn die Begriffe, die im Pschyrembel gebracht werden, können weitgehend 
von kostenlos zugänglichen Datenbanken ebenso aufgegriffen und verbreitet werden. Eine andere Situation ist beim Palandt-Kommentar zum BGB gegeben. Der Palandt veröffentlicht die Gerichtsurteile und die Gesetzestexte sowie den entsprechenden Kommentar. Die Gerichtsurteile und die Gesetzestexte kann jede Datenbank übernehmen. Der wissenschaftliche Kommentar, der auf allerhöchster wissenschaftlicher Ebene entsteht, ist urheberrechtlich geschützt und darf nicht übernommen werden. Die Absatzzahlen, die für den Palandt heute gelten, machen deutlich, dass es sich hier um ein derart gutes Produkt handelt, dass es auch weiterhin seinen kommerziellen Markt haben wird. Das Gleiche gilt für die Daten im Beilstein-Handbuch der organischen Chemie, das bei Elsevier verlegt wird und immer noch ein kommerzielles Erfolgsprodukt ist, weil die Informationen, die hier vermittelt sind, absolut originär entwickelt werden und entsprechend urheberrechtlich geschützt sind. Die entscheidende Frage ist, wie viel solcher inhaltlichen Werte erhalten oder neu geschöpft werden können, um das kommerzielle Modell weiter zu bedienen und auszulasten. Eine noch so vorsichtige Hochrechnung über die weitere Entwicklung macht deutlich, dass der Umsatzrückgang für die wissenschaftlichen Verlage in den nächsten Jahren eher 15 Prozent erreicht, als dass er unter 15 Prozent bleibt.

7) Ein Rückgang von 15 Prozent stellt die Verlage vor extreme Probleme. Das heißt, dass der Verlag auf allen Ebenen mit Gewinneinbußen oder Margen-Verschlechterungen rechnen muss. Es gibt Produkte, die auch heute noch einen Gewinn von 30 Prozent erreichen. Diese Gewinne werden sich aber bei den Produkten innerhalb der nächsten 10 Jahre auf 10-15 Prozent reduzieren. Produkte, die heute noch 3 oder 5 Prozent Gewinn erzielen, werden in wenigen Jahren nur noch mit Ergebnissen im negativen Bereich arbeiten können. Da die Verlage heute schon mit sehr niedrigen Gewinnmargen arbeiten, bedeutet dies in den meisten Fällen, dass eine Entwicklung um drei Prozent Reduktion der Gewinnmarge oftmals schon einen Verlust darstellt. Damit wird sich für zahlreiche Unternehmungen die Existenzfrage stellen. Auf der einen Seite wird diese Frage dadurch beantwortet, dass das Unternehmen an einen größeren Verlag verkauft wird. In anderen Fällen wird es aber zur Liquidation führen. Eine ganz entscheidende Frage für die Zukunft ist: Gelingt es den Verlagen, neue Produkte zu schaffen, von denen wir heute noch gar nichts wissen, die sie aber auf ihrem Markt verkaufen können? Einige Verlage haben seit vielen Jahren bereits damit begonnen,
Kongresse, Fortbildungsveranstaltungen und Symposien für ihre Klientel zu organisieren, die teilweise durchaus gewinnträchtig sind. Es gibt kommerziell verwertbare Projekte, wie das Beispiel der ApothekenUmschau zeigt, die die Verlage erfolgreich am Markt platzieren können.

\section{Vorhersage für 2020}

Die Pandemie hat natürlich auch beachtliche Auswirkungen auf die Entwicklung der Verlage in der Bundesrepublik Deutschland. Eine Umfrage ergab, dass die meisten Verlage mit Rückgängen zwischen 2 bis 15 Prozent rechnen. Im Bereich Fachzeitschriften einschließlich OnlineVerkauf bzw. Open Access dürften sich die Umsätze in etwa gegenüber dem Vorjahr noch halten, weil das Zeitschriftengeschäft immer im 1. Quartal des Jahres läuft, wo die Corona-Auswirkungen noch nicht so zu spüren waren.

\section{Ausblick für 2021}

Die Rückgänge im Jahr 2021 werden auf jeden Fall stärker sein als im Jahr 2020. Man rechnet hier mit Umsatzverlusten von 3 bis 10 Prozent, in einzelnen Fällen noch mehr, und es wird eine Reihe von Verlagen geben, die nicht mehr lebensfähig sind und die verkauft werden; aber das wird in vielen Fällen kaum noch möglich sein, da der am Kauf interessierte Verlag kaum noch in der Lage ist, die finanziellen Mittel aufzubringen, um in größerem Umfang Verlage hinzu zu kaufen, so dass eine Reihe von Verlagen liquidiert werden müssen.

Insgesamt sieht die Entwicklung ausgesprochen negativ aus und das Jahr 2021 wird ein ungewöhnlich schwieriges Jahr werden mit entsprechend negativen Ausblicken für die Zukunft.

\section{Anhang}

Die Entwicklung in den Bereichen Naturwissenschaften, Technik, Landwirtschaft sowie Rechtswissenschaften, Pädagogik/ Schulbuch

Tab. 3: Die Entwicklung der juristischen Verlage 2018-2019

\begin{tabular}{lll}
\hline Haufe, Freiburg & 330 Mio. & plus $10 \%$ \\
C. H. Beck, München & 320 Mio. & plus 8,6 \% \\
Wolters Kluwer & 277 Mio. & plus 3,4 \% \\
\hline
\end{tabular}




\begin{tabular}{lll}
\hline $\begin{array}{l}\text { Weka, Augsburg } \\
\text { Rentrop, Bonn-Bad }\end{array}$ & $\begin{array}{l}199 \text { Mio. } \\
\text { Godesberg }\end{array}$ & $\begin{array}{l}\text { minus 1,2 \% } \\
\text { minus 7,4 \% }\end{array}$ \\
Juris, Saarbrücken & 55,1 Mio. & plus 7,6 \% \\
NWB & 41,6 Mio. & Gleichstand \\
Otto Schmidt & 36,5 Mio. & minus 7,7 \% \\
Kohlhammer & 36,4 Mio. & plus 13,7\% \\
Boorberg & 30,3 Mio. & plus 2\% \\
Stollfuss & 25 Mio. & Gleichstand \\
Nomos, Baden-Baden & 21,5 Mio. & Gleichstand \\
Erich Schmidt & 19 Mio. & Gleichstand \\
Walhalla, Regensburg & 12,5 Mio. & Gleichstand \\
Bund-Verlag, Köln & 12 Mio. & Gleichstand \\
\hline
\end{tabular}

Im Jahr 2019 haben 15 juristische Verlage in Deutschland einen Umsatz von mehr als 12 Mio. Euro erreicht. Die Umsätze pro Verlag stiegen zwischen 13,7 Prozent für Kohlhammer, Stuttgart und minus 7,4 Prozent für Rentrop in Bonn-Bad Godesberg. Sechs Verlage hatten Steigerungen, sechs Verlage Stagnation.

Insgesamt stiegen die Umsätze um 94,3 Mio. Euro bei den juristischen Verlagen, das wäre ein Mittelwert von 6,9 Prozent. Damit machen die juristischen Verlage mehr als 15 Prozent des Gesamtumsatzes der deutschen Verlage aus.

Die Situation für die Verlage sieht wie folgt aus:

Tab. 4: Die Entwicklung der naturwissenschaftlichen, technischen \& landwirtschaftlichen Verlage 2019-2020

\begin{tabular}{|c|c|c|c|}
\hline Springer-Verlag & 590,1 Mio. & plus $2,4 \%$ & 14,2 Mio. \\
\hline Thieme & 161 Mio. & minus $0,9 \%$ & $\begin{array}{l}\text { minus } \\
1,5 \text { Mio. }\end{array}$ \\
\hline Wiley VCH & 138,6 Mio. & minus $0,4 \%$ & $\begin{array}{l}\text { minus } \\
555000\end{array}$ \\
\hline $\begin{array}{l}\text { Deutscher } \\
\text { Fachverlag }\end{array}$ & 134800 & $\begin{array}{l}\text { minus } \\
3,49 \%\end{array}$ & $\begin{array}{l}\text { minus } \\
457000\end{array}$ \\
\hline $\begin{array}{l}\text { De Gruyter, } \\
\text { Anteil }\end{array}$ & 15 Mio. & - & - \\
\hline Beuth & 78,4 Mio. & plus $5,4 \%$ & plus 4,2 Mio. \\
\hline $\begin{array}{l}\text { Deutscher } \\
\text { Apotheker- } \\
\text { Verlag }\end{array}$ & 69,1 Mio. & plus $0,9 \%$ & plus 622000 \\
\hline $\begin{array}{l}\text { Landwirt- } \\
\text { schaftsverlag }\end{array}$ & 55 Mio. & - & - \\
\hline $\begin{array}{l}\text { Deutscher } \\
\text { Ärzteverlag }\end{array}$ & 48,5 Mio. & minus $6,9 \%$ & $\begin{array}{l}\text { minus } 3,8 \\
\text { Mio. }\end{array}$ \\
\hline VDE & 46 Mio. & plus $1 \%$ & plus 460000 \\
\hline Hanser & 24 Mio. & - & - \\
\hline
\end{tabular}

\begin{tabular}{lcll}
\hline Ulmer & 28,9 Mio. & minus 2,9\% & $\begin{array}{l}\text { minus } \\
578000\end{array}$ \\
Rudolf Müller & 25,5 Mio. & minus 2,7\% & $\begin{array}{l}\text { minus } \\
690000\end{array}$ \\
$\begin{array}{l}\text { Handwerk und } \\
\text { Technik }\end{array}$ & 9,5 Mio. & minus 1\% & minus 190 \\
\hline & & & \\
\hline & 1434100 & plus 0,8 \% & plus \\
& Mio. & & 11712000 \\
\hline
\end{tabular}

Der Verlagsbereich hat nur noch ein minimales Plus von $0,8 \%$. Ein gewisses Plus ist einzig bei Springer und bei Beuth zu verzeichnen.

Die Verlage rechnen aber damit, dass sich 2020 ein Minus von 1 bis 2 Prozent im Schnitt ergibt und dass sich dieses Minus im Jahr 2021 eher noch erhöhen wird, da die Zeitschriftenumsätze im Jahr 2020 bereits im Frühjahr gebucht wurden und das war vor Ausbruch der Pandemie.

Tab. 5: Die Entwicklung der pädagogischen und Schulbuchverlage

\begin{tabular}{|c|c|c|c|}
\hline Klett & 330 Mio. & plus 3,7 \% & 12,2 Mio. \\
\hline Westermann & 300 Mio. & - & - \\
\hline Cornelsen & 250 Mio. & minus $2,9 \%$ & minus 5 Mio. \\
\hline Hueber & 32 Mio. & minus $5 \%$ & minus 1,6 Mio. \\
\hline $\begin{array}{l}\text { Bamberger } \\
\text { Verlagshaus }\end{array}$ & 21,3 Mio. & plus $4,4 \%$ & plus 940000 \\
\hline \multirow{2}{*}{$\begin{array}{l}\text { Europa Lehr- } \\
\text { mittel }\end{array}$} & 15,5 Mio. & plus $7 \%$ & plus 1090000 \\
\hline & 933315500 & plus $0,82 \%$ & plus 7630000 \\
\hline
\end{tabular}

Der Verlagsbereich Pädagogik/Schulbuchverlage hat im Jahr 2019 noch ein minimales Plus von 0,82 \%. Für das Jahr 2020 rechnet man mit einem Rückgang von 1 bis $2 \%$ und für das Jahr 2021 mit einer weiteren Erhöhung des Rückganges.

\section{Kommentar}

Die Entwicklung für Naturwissenschaften und verwandte Bereiche sowie für Pädagogik/Schulbuch fallen vergleichsweise negativ und stagnativ aus. Die einzig positive Ausnahme sind die juristischen Verlage, die laufend mit Ertragserhöhungen und Umsatzsteigerungen rechnen können. So wurde zum Beispiel bekannt, dass der Verlag C. H. Beck in München 2019 sein bisher bestes Ergebnis, was Umsatz und Ertrag anbetrifft, erreicht hat, und dass er davon ausgeht, dass das im Jahr 2020 noch einmal überschritten wird. 
Der entscheidende Grund, dass Jura so vergleichsweise gut dasteht, liegt daran, dass der juristische Markt noch permanent wächst. Die Zahl der Studenten der Erstsemester im juristischen Bereich ist jedes Jahr höher als im Vorjahr. Die Zahl der Mitarbeiter bei Gerichten, in der Verwaltung und bei Behörden sowie bei Rechtsanwälten steigt ebenfalls permanent an und führt zu höchsten Gehältern, die es überhaupt gibt.

Der zweite entscheidende Grund, warum die juristischen Verlage so gut dastehen, liegt daran, dass es ihnen gelungen ist, die Online-Verwertung weitgehend kostenpflichtig zu halten. Das heißt, es werden kaum juristische Datenbanken im Open Access kostenlos verbreitet, denn die Online-Verbreitung ist in den meisten Fällen bei den Verlagen geblieben und nicht an behördliche oder institutionelle Großunternehmen gegangen, die für die Verbreitung der Daten sorgen. So hat zum Beispiel für C. H. Beck der Bereich Online einen Anteil von über 25 Prozent an den Erlösen. Der Bereich Online setzt sich bei Beck auf der einen Seite zu etwa 70 bis 75 Prozent aus Erlösen aus verlagseigenen Projekten, wo alle Rechte beim Verlag liegen, zusammen und rund 25 Prozent aus der Lizenzierung von Datenbeständen aus anderen Verlagen, die häufig zu klein sind, um einen entsprechenden Online-Vertriebsapparat aufziehen zu können, wie er bei Beck beispielsweise perfekt beherrscht wird. Das Gleiche gilt auch für Juris in Saarbrücken, das als Online-Verlag sehr erfolgreich ist.

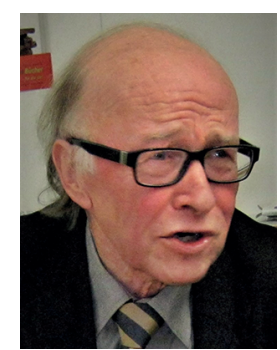

Prof. Dr. h. c. mult. Klaus G. Saur Beuerberger Straße 9 D-81479 München kgsaur@googlemail.com 